\title{
Performance Evaluation of Multiple-Rate Mobile Ad Hoc Networks ${ }^{1}$
}

\author{
Gang Ding ${ }^{+}$, Xiaoxin $\mathrm{Wu}^{*}$, and Bharat Bhargava* \\ ${ }^{+}$School of Electrical and Computer Engineering, Purdue University, W. Lafayette, IN 47907 \\ * Department of Computer Sciences, Purdue University, W. Lafayette, IN 47907 \\ Email: dingg@ecn.purdue.edu,wu@cs.purdue.edu,bb@cs.purdue.edu
}

\begin{abstract}
Existing wireless networks usually provide multiple data transmission rates. This paper presents a simulation study on the performance of multiple-rate mobile ad hoc networks (MANETs), based on an evolved ns-2 simulator. At the physical layer, realistic models such as Walfisch-Ikagami radio propagation model and lognormal fading are implemented. At the link layer, a link adaptation algorithm is implemented to select an appropriate data transmission rate based on the receiving signalto-noise ratio. At transport and application layers, different data traffics, including constant bit rate, TCP, voice over IP, and video, are generated. We study the network performance such as throughput, delivery ratio, and end-to-end delay when position-based routing is used. We also study how node mobility and position error affect the performance. In addition, we investigate the impact of the link distance, namely the geographic distance for a hop, on the end-to-end network throughput. This work is a comprehensive simulation study on the impact of various factors on the performance of MANETs. It also provides guidelines for future protocol and algorithm design.
\end{abstract}

Keywords: mobile ad hoc network, link adaptation, link distance, network allocation vector.

\footnotetext{
${ }^{1}$ This work is partly supported by NSF grants ANI 0219110 and CCR 0001788, and Motorola Labs.
} 


\section{INTRODUCTION}

Mobile ad hoc networks (MANETs) have been widely studied in the literature [1-3]. Due to the nature of self-organization, the dynamic topology caused by mobility and transmission power control, and the multiple-hop routing in MANETs, it is difficult to build a complete analytical model to study the network performance. On the other hand, a real testbed is expensive. Therefore, the simulation study of MANETs is important. Different simulation tools, such as ns-2 [4] with CMU monarch extension [5], GloMoSim [6] and its commercial successor QualNet [7], OPNET [8], and SWANS [9], have been developed for MANET evaluation. The simulation study presented in this paper is based on ns-2 because it is open source and is widely used in both academia and industry.

In summary, ns-2 has following features:

1) For radio propagation, the Friss-space model is used for short distances and the approximated two-ray-ground model is used for long distances. The shadowing model [21] is employed to characterize the probabilistic multiple path fading during radio propagation. There are some other extensions to ns-2, for example, Ricean fading [22] and accurate physical layer modeling [23].

2) At MAC layer, the IEEE 802.11 distributed coordination function (DCF) [10] is implemented, including Request-to-Send (RTS) / Clear-to-Send (CTS) / DATA / ACK four-way handshake for unicasting packets.

3) At network layer, major ad hoc routing protocols, such as Destination Sequence Distance Vector (DSDV) [11], Ad hoc On-demand Distance Vector (AODV) [12], and Dynamic Source Routing (DSR) [13], are implemented.

4) At transport and application layers, random connections of Constant Bit Rate (CBR) and TCP data traffics can be generated by a traffic-scenario generator. 
5) The random way-point mobility model is developed, which is specified by the maximum speed of movements, the pause time between movements, and the direction of the movements.

Previous simulation studies on MANETs mainly focused on routing protocols and their scalability issues $([7,9])$. In this paper, however, we investigate the impacts of different factors at different layers on the system performance. It is noted that the CMU monarch extension to ns-2 models the Lucent Wavelan card at a fixed data rate [5]. But we will investigate the performance of a multiple-rate MANET supported by current IEEE 802.11 standards. Specifically, we explore the data delivery ratio, throughput, transmission delay, and hop count in a MANET with multiple data transmission rates available at the physical layer.

To conduct the above-mentioned simulation studies, we extend the current ns-2 in the following ways:

1) A more realistic urban area radio propagation model, along with lognormal fading model, is implemented.

2) To efficiently utilize the network bandwidth, a rate control algorithm is developed in the link layer so that a sender can select the highest available transmission rate based on the receiving SignalNoise-Ratio (SNR).

3) In addition to CBR and TCP data traffics, Voice over IP (VoIP) and streaming video traffic models are developed at application layer.

4) A position-based routing protocol GPSR [17] is developed, because it has been proven to achieve the highest throughput among the existing ad hoc protocols. Therefore, the performance in a network that uses position-based routing protocol can be regarded as an upper bound for the performance of MANETs. 
Accordingly, the major experiments we have conducted in this study are listed as follows.

1) When multiple data rates are available, we examine the network performance improvement when rate control algorithm is applied to the link layer. We study different Network Allocation Vector (NAV) estimation algorithms, and evaluate the impact of carries sense threshold.

2) The smaller hop count for a route implies larger link distance in each hop and consequently lower available link data rate. Therefore, routes with minimum hop counts (i.e., largest link distance) may not lead to the best end-to-end routing performance. We compare network performance where routes are determined under different link distance requirements and try to discover the relationship between the link distance and the end-to-end throughput.

3) Other than CBR data traffic, we examine the performance of MANET when TCP data traffic and real-time data traffics such as voice and video are applied to the transport and application layers.

4) Mobility is one of the major factors that affect the MANET performance. In position-based routing, position accuracy determines whether the correct routes are selected. Experiments are conducted when nodes are moving at different speeds or under different position errors.

The rest of the paper is organized as follows. Section II details the extension to ns-2 and the simulation scenario. Section III presents the simulation results for the link adaptation in MANETs, along with implementation details. Section IV explores the impact of link distance on the end-to-end throughput of multiple-rate MANETs. Performance of the systems involving various traffic patterns is studied in section V. The impact of position error and moving speed is studied in section VI. Section VII concludes the paper. 


\section{Simulation Setup}

\subsection{Radio Propagation Model}

The Walfisch-Ikegami model [14] is adopted to approximate the radio propagation between isotropic antennas. It shows a good fit to the real data measured in urban environments and it is regarded as one of the most accurate empirical models for the outdoor radio propagation. It has been recently used in the study of mobile wireless networks $[28,29]$. In contrast to only considering a flat area in the traditional two-ray-ground model, the Walfisch-Ikegami model considers the effect due to streets and buildings between the transmitter and the receiver. We have implemented the WalfischIkegami model at the physical layer in ns-2. Some parameters for the model are listed in Table 1 . They characterize the distance between buildings, the average heights of the building, transmitter and receiver, the transmission frequency, and so on. The details of the model are given in the Appendix.

Table 1. Parameters for Walfisch-Ikagami model

\begin{tabular}{||l|l|l||}
\hline Description & Parameter & Typical value \\
\hline Width of the street that separates buildings & $w$ & $25 \mathrm{~m}$ \\
\hline Distance between buildings & $b$ & $150 \mathrm{~m}$ \\
\hline Average building height & $h_{\text {roof }}$ & $30 \mathrm{~m}$ \\
\hline Transmitter height and receiver height & $h_{l}$ and $h_{2}$ & $1-50 \mathrm{~m}$ \\
\hline Distance between transmitter and receiver & $d$ & $20-5000 \mathrm{~m}$ \\
\hline Propagation angle between the radio path and street & $\varphi$ & 55 degrees \\
\hline Transmission frequency & $f$ & $2.4 \mathrm{GHz}$ \\
\hline
\end{tabular}

Current implementation of ns- 2 does not consider the packet loss and channel fading during radio propagation. We add a packet loss model in ns-2 which includes the lognormal fading [21]. The 
lognormal fading has a standard deviation of $6 \mathrm{~dB}$ and $10 \mathrm{~dB}$ corresponding to suburban and urban environments, respectively, and a correlation coefficient of 0.5 .

\subsection{Link Adaptation}

Since multiple data transmission rates are available in many wireless communication systems, how to select an appropriate data rate is an interesting research topic. There have been many results on link adaptation at link layer to choose the data transmission rate at physical layer. [24] theoretically analyzed the effect of link adaptation on IEEE 802.11a WLAN. They chose sender based algorithm in which the transmitter decides which rate to use based on the wireless channel condition around the sender. But the sender based approach cannot guarantee that the receiver can successfully receive the packet because the transmitter does not know the network condition at the receiver's side. Some control packets can be exchanged between the transmitter and the receiver to learn the information at both ends of the transmission link, but this introduces too much overhead and has to modify the current standard. So many new link adaptation schemes are based on the receiver. [25], [26], and [27] proposed the link adaptation algorithms conducted by the receiver in IEEE 802.11 WLAN, GPRS, and HiperLAN/2, respectively. In this paper, we implement a receiver based link adaptation algorithm [15] to decide the data transmission rate. Upon receiving the RTS, the receiver makes estimation of SNR and selects an appropriate data rate according to Table 2. We adopt IEEE 802.11g standard [16] which provides multiple data rates by various Extended Rate PHY (ERP) modulation modes at physical layer. The information about the selected rate is inserted to CTS and sent back to the sender. The sender will use this rate for the following data transmission. RTS and CTS packets themselves are transmitted at the basic data rate of $1 \mathrm{Mb} / \mathrm{s}$. This scheme is implemented as an extension to the standard channel access mechanism provided by ns-2. 
Table 2. Data rate selection

\begin{tabular}{|c|c|c|}
\hline SNR $(d B)$ & Data rate $(M b / s)$ & \multirow{2}{*}{ Modulation mode } \\
\hline$>30$ & 48 & \multirow{2}{*}{ ERP-OFDM } \\
\hline $26-30$ & 36 & \multirow{2}{*}{ ERP-CCK } \\
\hline $21-26$ & 24 & \multirow{2}{*}{ ERP-DSSS } \\
\hline $18-21$ & 11 & \\
\hline $16-18$ & 5.5 & \\
\hline $14-16$ & 2 & \\
\hline $11-14$ & 1 & Packet loss \\
\hline$\leq 11$ & \multicolumn{2}{|c|}{} \\
\hline
\end{tabular}

One problem with the above rate selection scheme is that it is impossible for the sender to include the precise transmission duration time in RTS, which is required by the IEEE 802.11 standard [10]. This is because that, at this moment, the sender does not know exactly what data rate will be used. Yet the duration time determines NAV, which plays an important role for better CSMA-CA performance. We propose several ways to determine NAV, which will be explained and tested in the next section.

\subsection{Position-Based Routing}

The routing protocols have been the major topic of previous study on MANETs, but in this paper we are more interested in the performance due to other system components. To reduce the effect due to a particular routing protocol, we implemented a position-based protocol [17] which assumes that the position information of each mobile node is available so that the routing decision is only based on the positions of source, destination and neighbor nodes. 


\subsection{VolP and Video Data Traffic}

For VoIP data traffic, the constant rate data flow with an interval of $20 \mathrm{~ms}$ between any two consecutive data packets is used. The packet size is 20 bytes. For video data traffic, MPEG-4 video trace data is obtained from a 60-minute movie the Jurassic Park, which is publicly available for the test of video transmission [18], especially for wireless networks [19]. A video data traffic generator is developed so that the trace data are fragmented with a given packet unit size and transmitted at a given frame rate. Some important parameters for video data traffic are given in Table 3.

Table 3. Parameters for video data traffic

\begin{tabular}{||l|l||}
\hline Parameter & Value \\
\hline Resolution & QCIF $176 \times 144$ \\
\hline Frame rate & 25 frames $/ \mathrm{sec}$ \\
\hline Frame sequence & IBBPBBPBBPBB \\
\hline Compression ratio & YUV: 49.96 \\
\hline Video run time & $3.6 \mathrm{e}+6 \mathrm{msec}$ \\
\hline Min frame size & 26 bytes \\
\hline Max frame size & 8154 bytes \\
\hline \hline
\end{tabular}

\subsection{Network Scenario}

Without other specification, the ad hoc network studied in this paper involves 100 nodes uniformly distributed in a square area of $1000 \mathrm{~m} \times 1000 \mathrm{~m}$. Each node moves within the area, with a random direction and a random velocity uniformly distributed between 0 and a maximum value of $10 \mathrm{~m} / \mathrm{s}$. When data is transmitted at $1 \mathrm{Mb} / \mathrm{s}$, i.e., the basic data rate, a receiver can successfully receive the data 
when it is as far as $250 \mathrm{~m}$ away from the sender. For a connection between a source and a destination, the default data traffic is CBR with four packets per second and 512 bytes per packet.

\section{SIMULATION ON LINK ADAPTATION}

In this section, we first show the performance improvement when data rate control algorithm is applied in the link layer. Then two implementation issues related to NAV and carrier sense threshold are addressed in details.

\subsection{Performance Improvement by Rate Control}

We first compare the system performance between the rate control case and the case when there is no rate control so that the default fixed transmission rate is used. Figure 1 shows the packet delivery ratio, transmission delay, throughput, and hop count, before and after the rate control algorithm is applied. The load of network traffic is varying from 10 to 50 connections. It is shown that, after the rate control algorithm is applied, the delivery ratio is improved from $69 \%$ up to $171 \%$, the transmission delay is reduced from $21 \%$ to $96 \%$, and the throughput is improved from $71 \%$ to $165 \%$. The hop counts in both cases are very close to each other because they use the same routing protocol to find the shortest path.

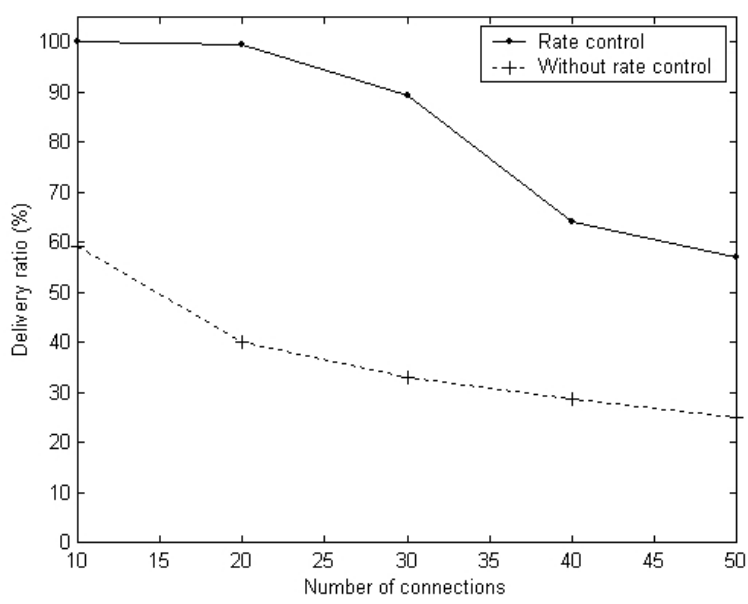

a. Delivery ratio

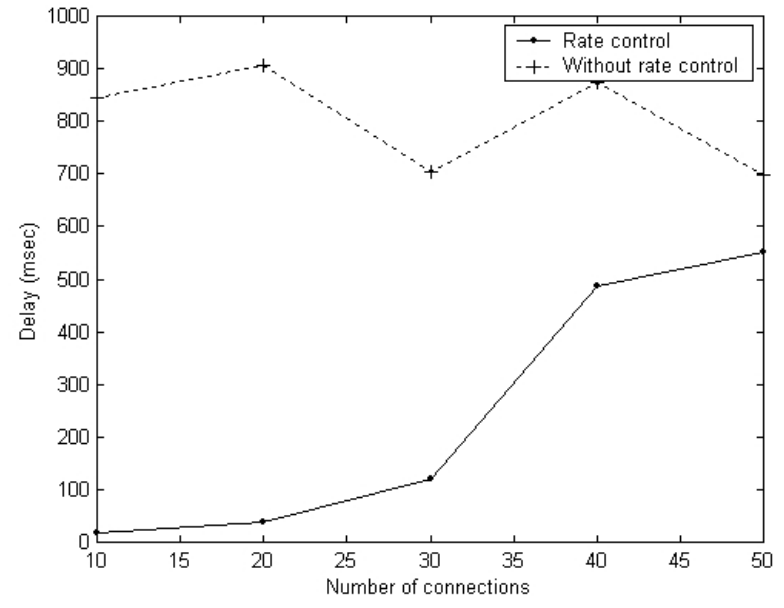

b. Transmission delay 


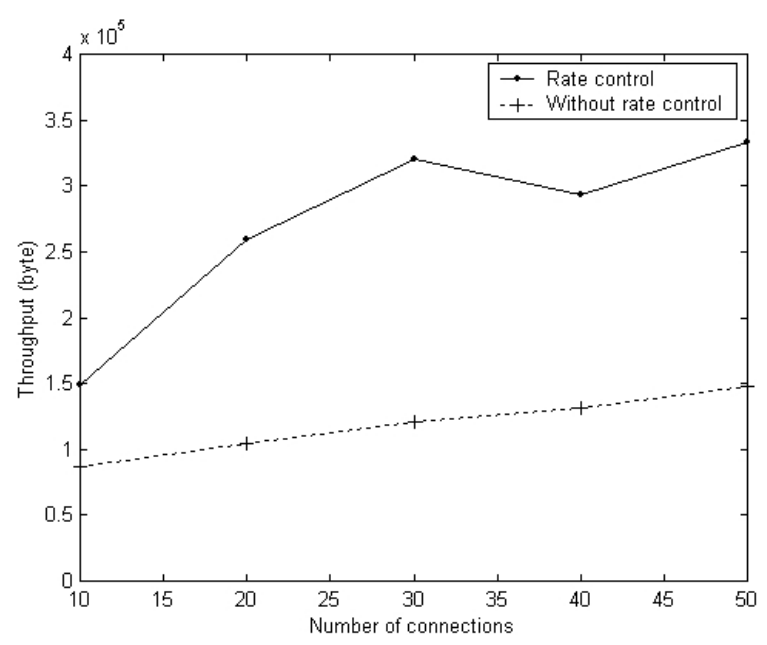

c. Throughput

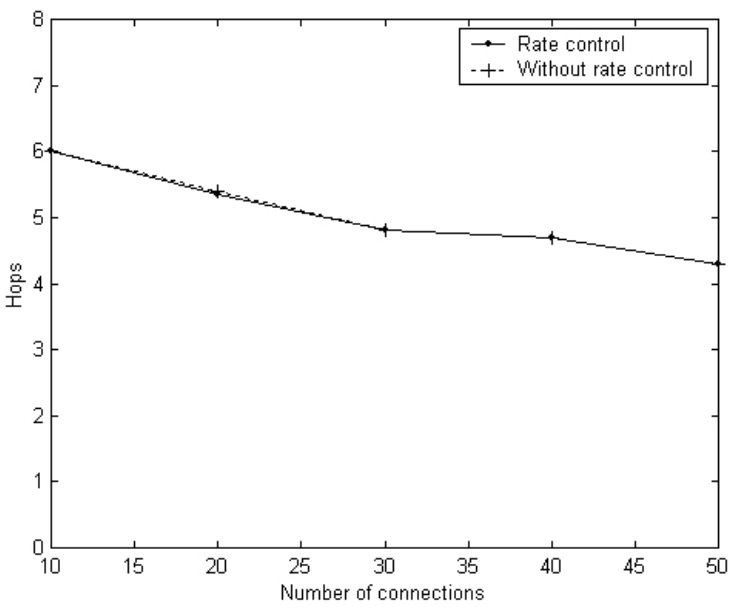

d. Hop count

Figure 1. Results of rate control

Figure 2.a shows the data rate dynamically selected by each sent packet during the first 10 seconds of simulation. The density of points for each rate represents how often this rate is selected. The total number of packets transmitted at different rates is given in Figure 2.b. The average of selected data rate is shown in Figure 2.c. Which rate is selected largely depends on the network traffic and topology. According to Figure 2, when more connections, that is more network traffic, are involved, a smaller data rate tends to be selected because more interferences from nodes in the neighborhood attenuate the quality of received data. This also explains why the transmission delay increases when the number of connections increases because smaller data rate implies longer transmission time.

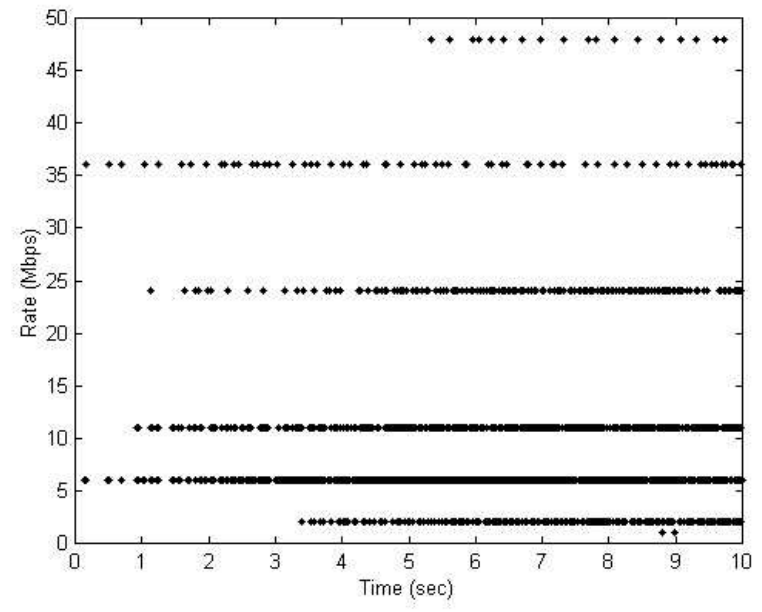

a. Data rate histogram

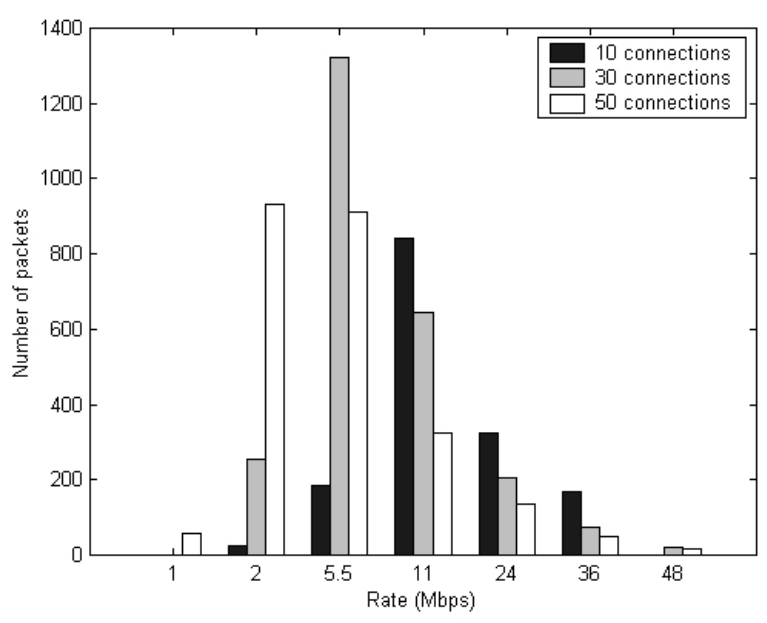

b. Number of packets sent at different rates 


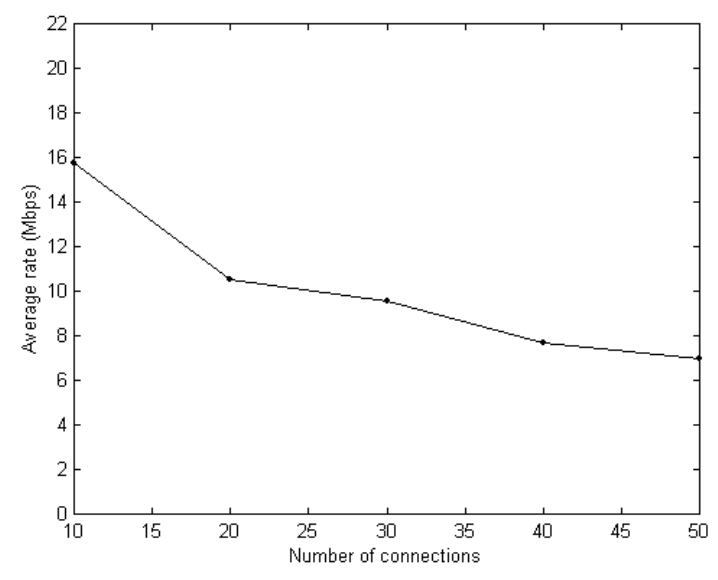

c. Average data rate

Figure 2. Statistics of selected data rate

\subsection{NAV estimation and update}

At IEEE 802.11 MAC layer, the NAV used in RTS and CTS packets indicates the time period during which other mobiles nodes can not access the shared wireless channel. NAV in RTS and CTS is calculated as shown in Figure 3, where SIFS represents the Short InterFrame Space that a packet must wait before transmission. Since RTS, CTS and ACK are all transmitted at the basic rate, NAV can be easily calculated if the transmission rate of the DATA packet is fixed and known before RTS and CTS are sent. However, when the above adaptive rate control algorithm is applied, the actual data rate is selected by the receiver upon receiving the RTS. This implies that the NAV can only be estimated in RTS by the sender. It can be updated later in CTS by the receiver, based on the actual selected data rate. But in the real system, a neighbor who receives an RTS and sets an NAV accordingly may not be able to receive the following CTS with an updated NAV. So it may not always be beneficial to take extra effort to update the NAV in CTS. 

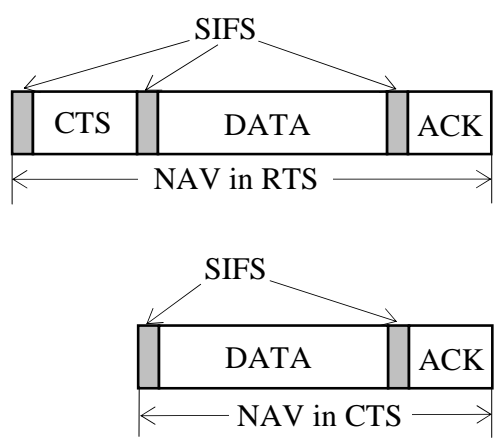

Figure 3. NAV in RTS and CTS

There are many ways to estimate NAV in RTS and update it in CTS. We here compare five different schemes.

- NAV 1: Uses the maximal transmission rate $54 \mathrm{Mb} / \mathrm{s}$ to estimate NAV in RTS, but does not update NAV in CTS.

- NAV 2: Uses the basic transmission rate $1 \mathrm{Mb} / \mathrm{s}$ to estimate NAV in RTS, but does not update NAV in CTS.

- NAV 3: Uses $54 \mathrm{Mb} / \mathrm{s}$ to estimate NAV in RTS and update NAV in CTS based on the selected new data rate.

- NAV 4: Uses the last data sending rate to estimate NAV in RTS, but does not update NAV in CTS.

- NAV 5: Assuming the actual data rate is known before sending the RTS, the appropriate NAV can be obtained in RTS so that it is not necessary to update it in CTS. This is the best but unrealistic case because the actual data rate selected by the receiver can not be known in advance by the sender when it is sending the RTS. We only use this scheme for comparison with others. 


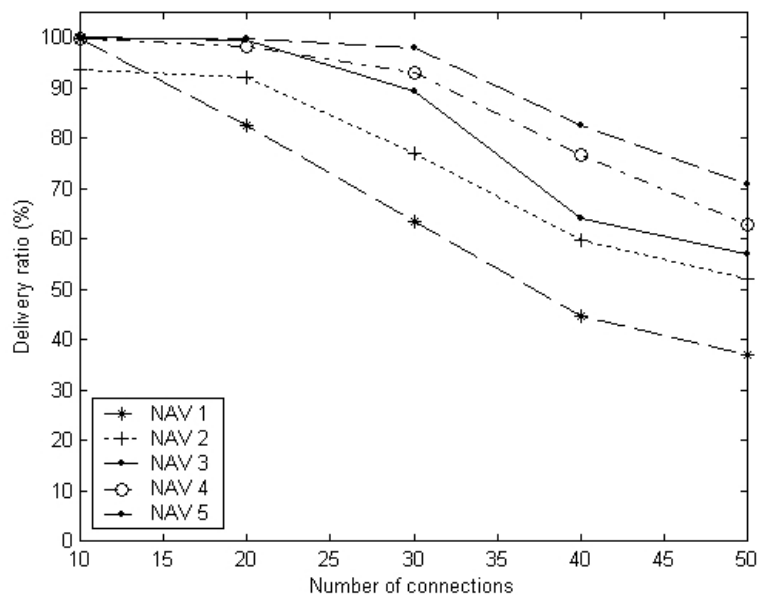

a. Delivery ratio

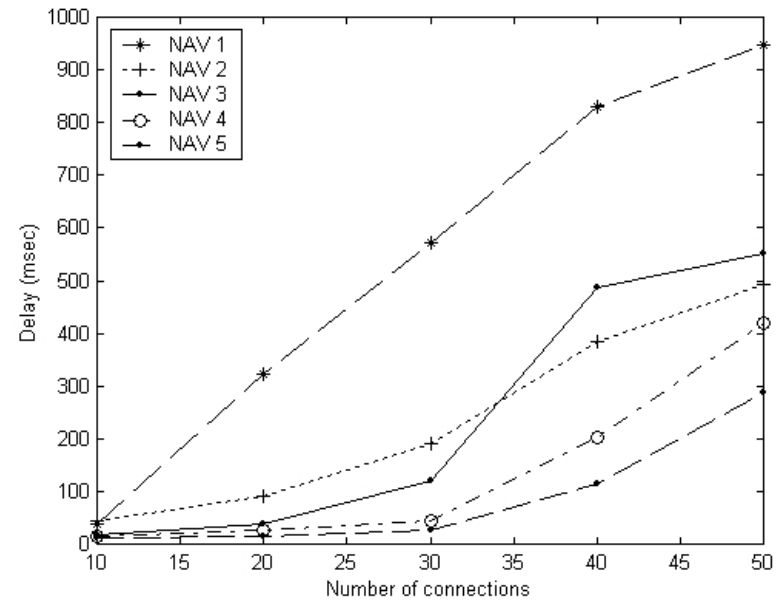

b. Transmission delay

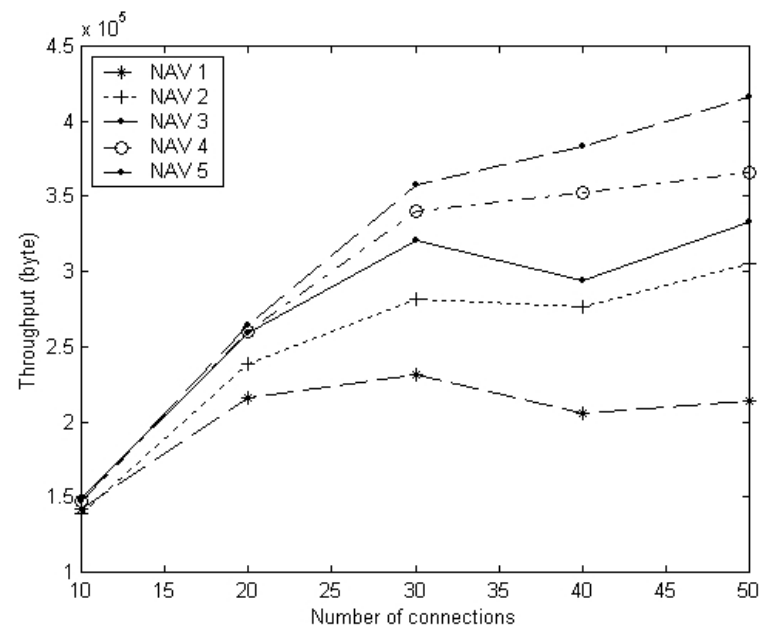

c. Throughput

Figure 4. Results of using different NAV schemes

For all the above schemes, Figure 4 shows the delivery ratio, transmission delay, and throughput. It is evident that, for most traffic loads from 10 to 50 connections, the performance in increasing order is NAV $1<$ NAV $2<$ NAV $3<$ NAV $4<$ NAV 5 . The only exceptions are that the delivery ratio of NAV 3 and NAV 1 outperform NAV 4 and NAV 2, respectively, at low traffic loads; and the transmission delay of NAV 3 is a little larger than NAV 2 for high traffic loads. According to these simulation results, we observe that small transmission rate is preferred for NAV estimation in RTS because this yields longer NAV so that less collision and interference will happen during the real data transmission. We also observe that, when NAV in CTS is updated as in NAV 3, it works better when the traffic load 
is low. Since NAV 3 and NAV 4 perform better than NAV 1 and NAV 2 in most cases, NAV 3 and NAV 4 are recommended. NAV 4 performs better for high traffic loads and is simpler because it does not need to update NAV in CTS. But NAV 3 performs better for low network loads. We will use NAV 3 in the following simulations.

\subsection{Carrier Sense Threshold}

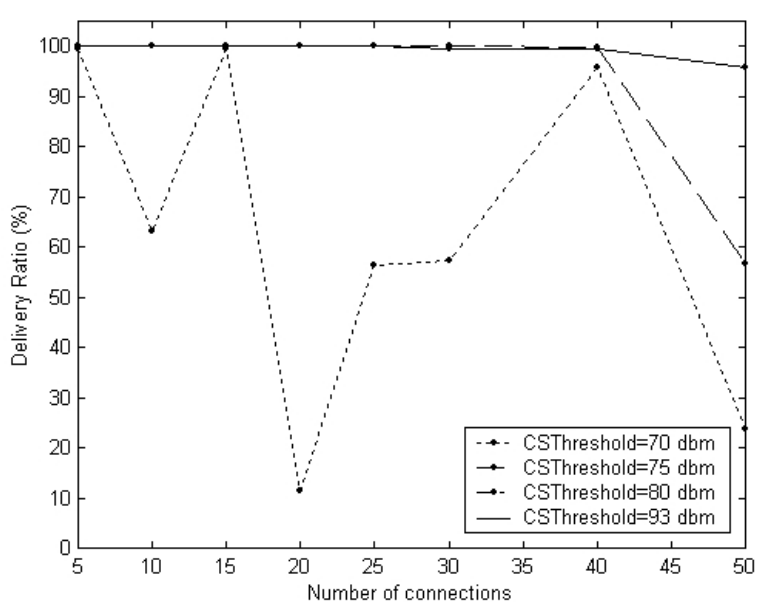

a. Delivery ratio

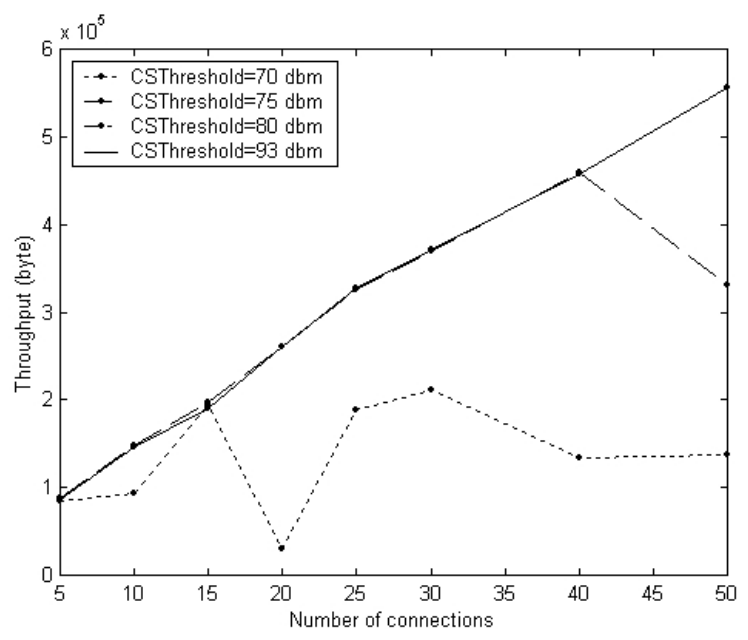

c. Throughput

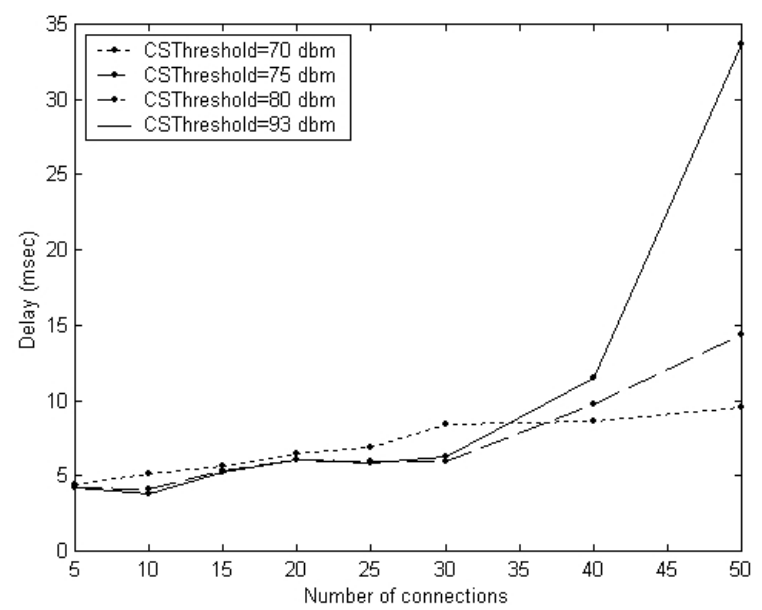

b. Transmission delay

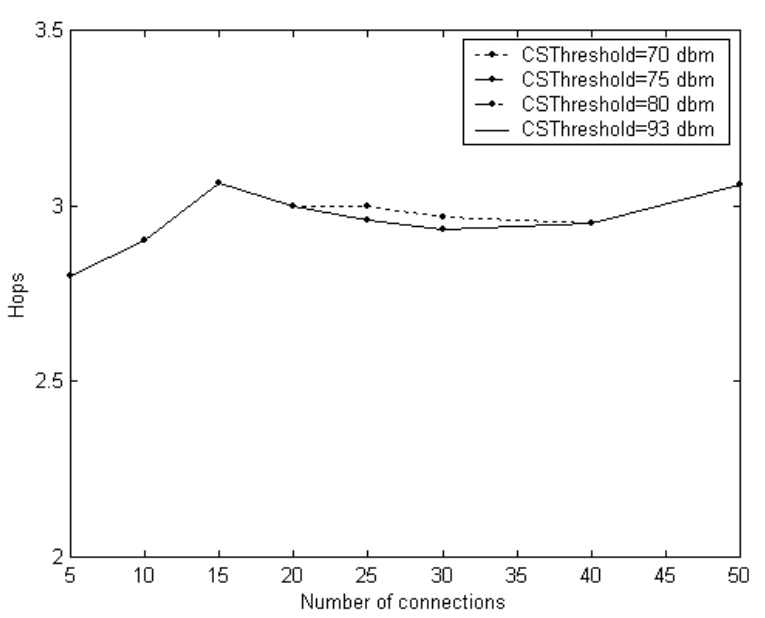

d. Hop count

Figure 5. Results of using different carrier sense thresholds

Carrier Sense Threshold (CSThresh) decides if an arriving packet has enough energy to be detected and correctly decoded. It is an important parameter at physical layer that affects the performance of upper layers. We compare the system performance due to different carrier sense thresholds at 70, 75, 
80 , and $93 \mathrm{dBm}$. The delivery ratio, transmission delay, throughput, and hop count are given in Figure 5. It is obvious that higher CSThresh maintains higher delivery ratio and throughput. But if it is as low as $70 \mathrm{dBm}$, a large percent of packets can be lost, which is supported by the unsmooth curves of delivery ratio and throughput in Figure 5.

For low traffic loads, the transmission delays of different CSThreshs are very close. But when the number of connections increases, higher CSThresh shows higher transmission delay. This is due to the fact that only the received data packets are employed to calculate the average transmission delay. Since the delivery ratio for lower CSThresh is low at high network loads, there is actually a smaller number of packets being used for calculation of transmission delay. So we could get a smaller average delay for lower CSThresh. If we set the transmission delay of all the lost packets to be a large value, the corresponding average delay will be actually very high.

\section{Simulation ON LiNK Distance}

For most mobile ad hoc routing protocols, the hop count is a widely used metric. The route with the minimum hop count is preferred. This works well when the transmission data rate is fixed, because selecting the route with the minimum number of hops renders less active nodes in a network so that each node has a better chance to access the shared wireless channel, which results in a higher throughput. But when multiple data rates are available, the route with the minimum hop count may not result in the best end-to-end throughput. The reason is that a route with a smaller number of hops implies the longer geographic distance for each hop, and consequently a lower SNR at the receiving end as well as a lower available data rate according to Table 2. On the other hand, if we reduce the geographic distance of hops in order to achieve a high data rate, more intermediate nodes are needed for a route. Each node then has less opportunity to access the wireless channel. The achievable data rate and the number of active nodes are two contradictory factors that affect the overall network 
throughput.

In this section, for a multiple-rate ad hoc network, we investigate how the throughput is affected by routes built up by hops of different geographic distances. We call the geographic distance between a sender and its immediate next hop the link distance. Since it is difficult to find the hops that have exactly the required link distance, we set a maximum link distance, called communication range. Among all the neighbors within the communication range of the sender, the neighbor of the longest link distance is selected as the next hop.

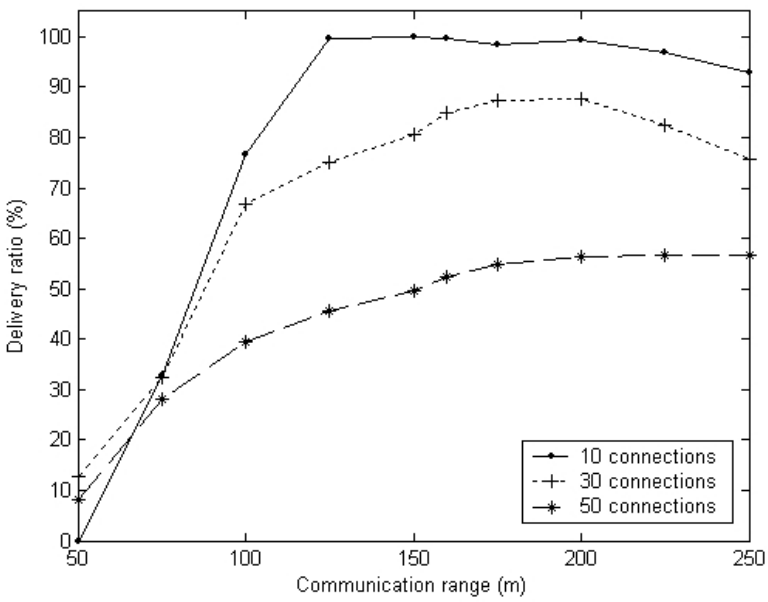

a. Delivery ratio

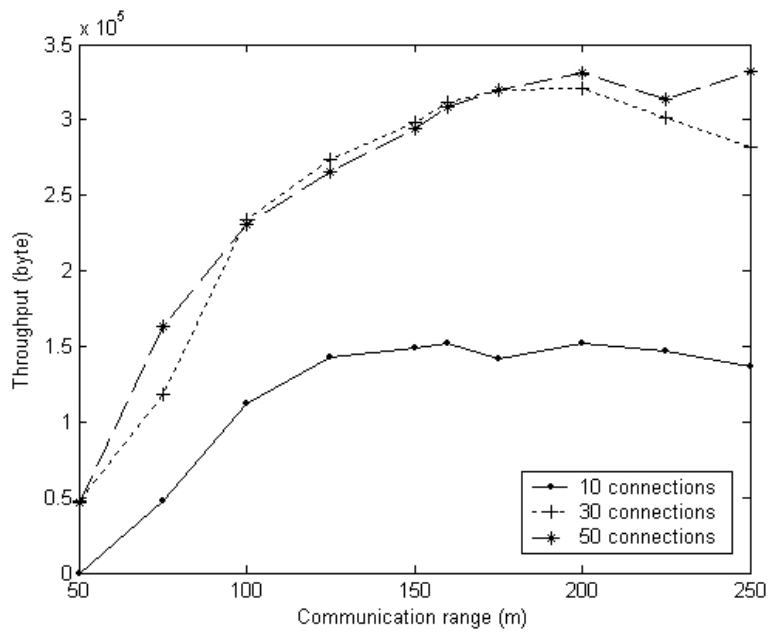

c. Throughput

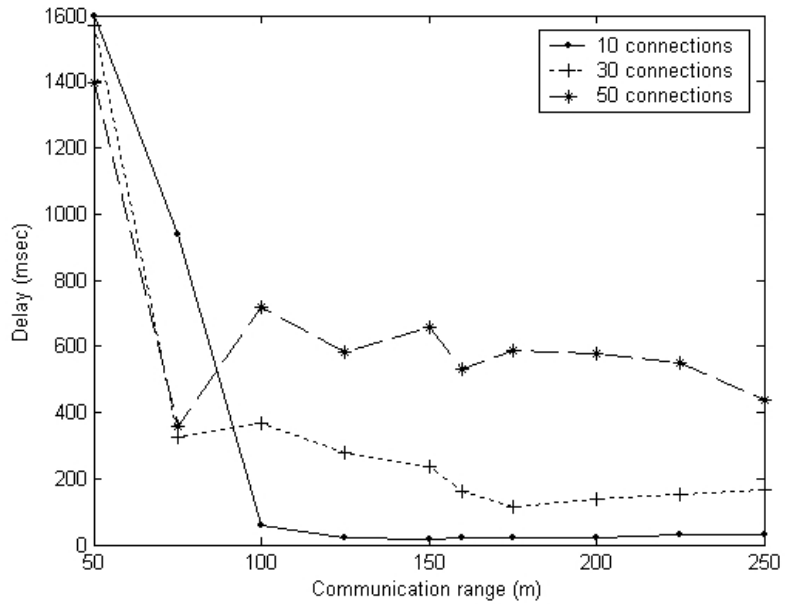

b. Transmission delay

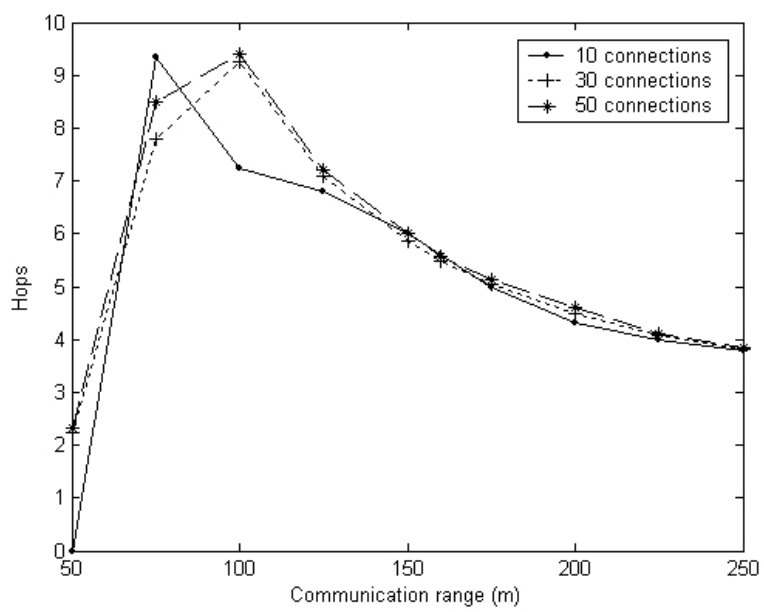

d. Hop count

Figure 6. Results of link distance 
Figure 6 shows the delivery ratio, transmission delay, throughput, and hop count when different communication ranges are used. The network with a light load of 10 connections, a medium load of 30 connections, and a heavy load of 50 connections are compared. The figure shows that there exists a communication range at which the highest delivery ratio is achieved. When the number of connections is small, the optimum communication range is small because fewer nodes will contend for the channel. Table 4 shows the optimum communication range at different network loads, resulted from extensive simulations. It is evident that the optimum communication range increases when the traffic load increases.

Table 4. Optimum communication range

\begin{tabular}{||l|c|c|c|c|c||}
\hline Number of connections & 10 & 20 & 30 & 40 & 50 \\
\hline Optimum communication range $(m)$ & 150 & 175 & 185 & 200 & 210 \\
\hline
\end{tabular}

Figure 7 compares the performance of systems using the optimum communication range and the fixed maximum communication range. Using maximum communication range corresponds to the case when the minimum hop count is preferred. As shown in Figure 7.d, the hop count of using optimum communication range is larger than that using the maximum communication range, but higher delivery ratio and throughput are achieved as in Figure 7.a and 7.c. According to Figure 7.b, reducing the communication range from its maximum value may not result in a longer end-to-end delay. The reason is because when the link distance decreases, although more transmissions are needed for a packet to be successfully delivered to the destination due to the increased hop count, the link distance in a hop gets shorter, so the data rate of the link is higher and the transmission time over the link is smaller. The overall transmission delay thus does not necessarily increase. Reducing the link distance generates routes with larger hop count (i.e., more contending nodes) but higher link data rate. This two-fold effect is further studied by analysis in [20], where an adaptive link distance algorithm is proposed to 
dynamically adjust the link distance to approximate the optimum communication range.

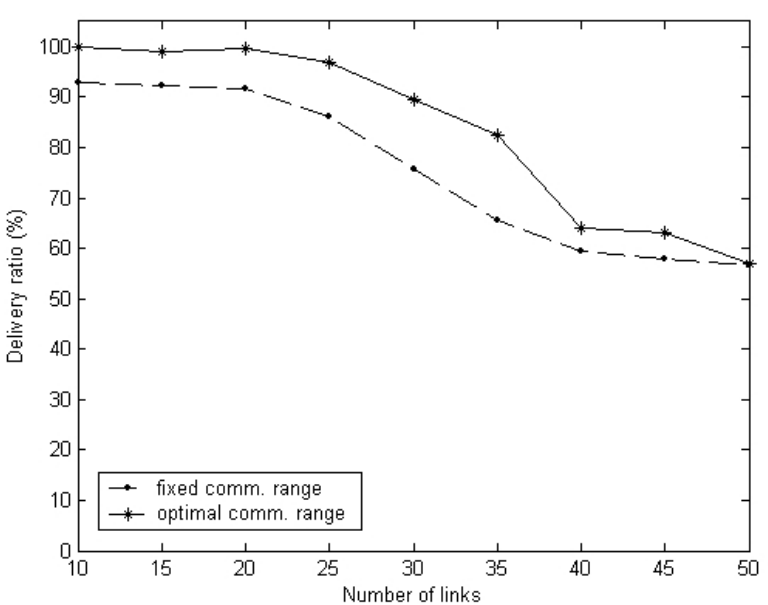

a. Delivery ratio

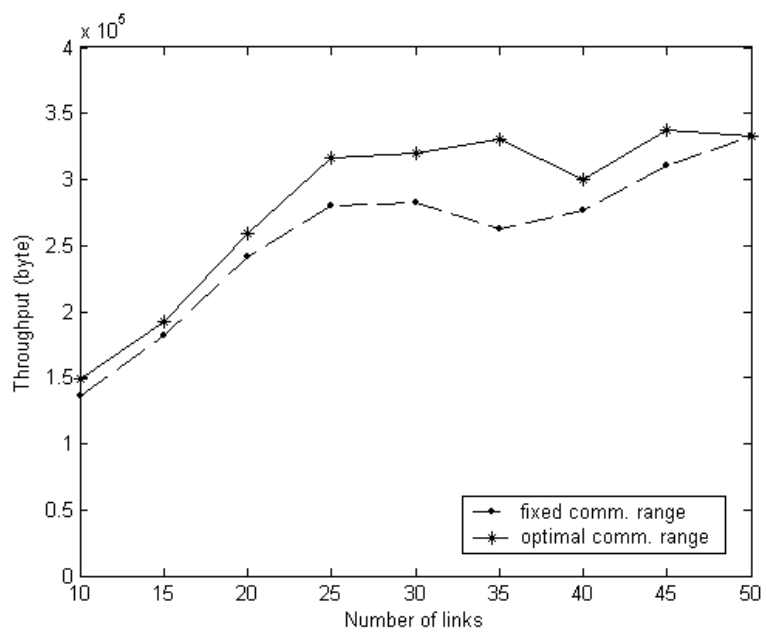

c. Throughput

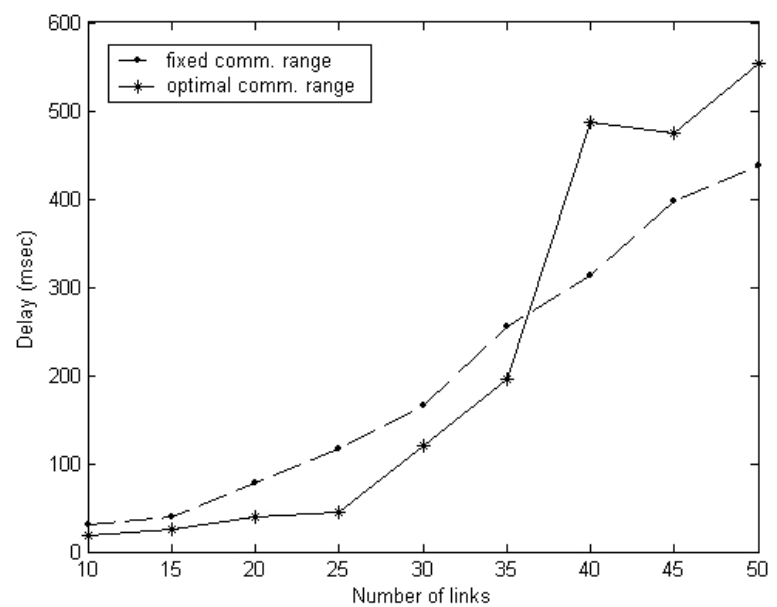

b. Transmission delay

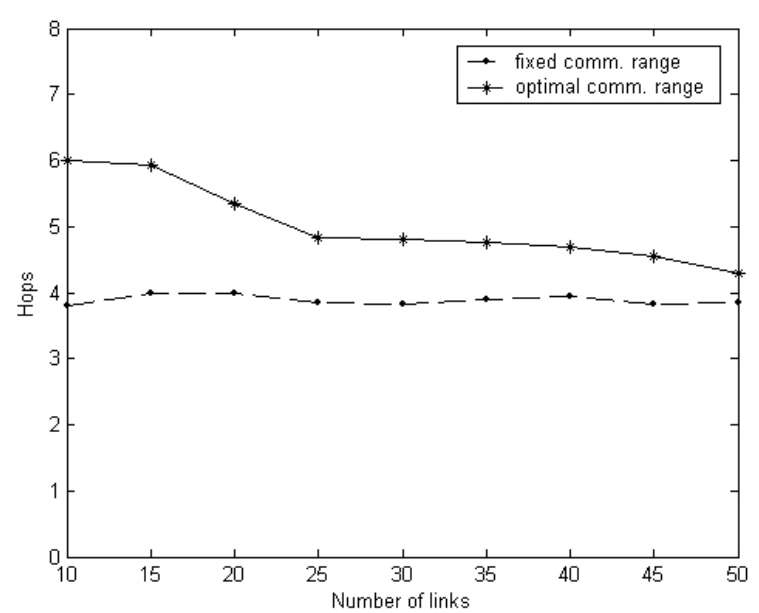

d. Hop count

Figure 7. Results of using optimum communication range

\section{Simulation on Data Traffic}

Previous sections use CBR data traffic and UDP transport control, the impact of other widely used data traffics, such as TCP, VoIP and Video, will be studied in this section.

\subsection{TCP traffic}

TCP traffic sends continuous data packets controlled by the flow and congestion control algorithms provided by TCP at transport layer. The simulation results are given in Figure 8 . TCP traffic can 
maintain a very good data delivery ratio: at least $95 \%$ of sent packets can be successfully received. The delivery ratio only drops a little for higher number of connections. But the transmission delay of TCP traffic is quite long. This is due to the flow and congestion control that scarifies the transmission time to the reliable transport. The transmission delay for TCP traffic is long even when the traffic load is low. This is due to the fact that higher data rate is chosen for low loads, which may introduce congestion at the intermediate nodes and trigger the TCP congestion control mechanism. As a result, the data transmission is delayed.

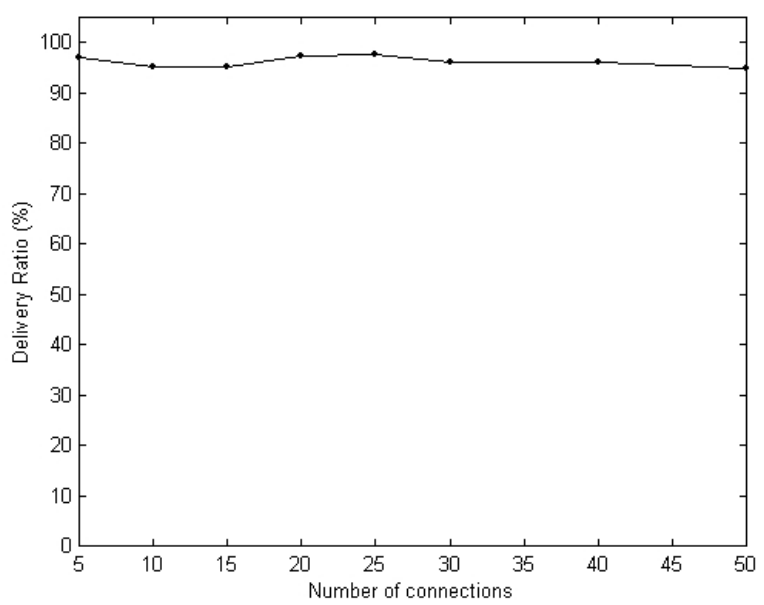

a. Delivery ratio

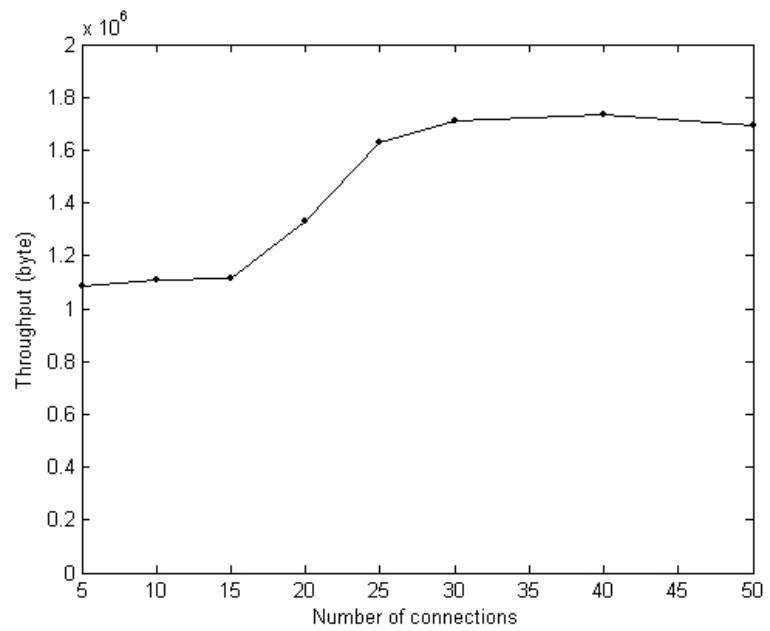

c. Throughput

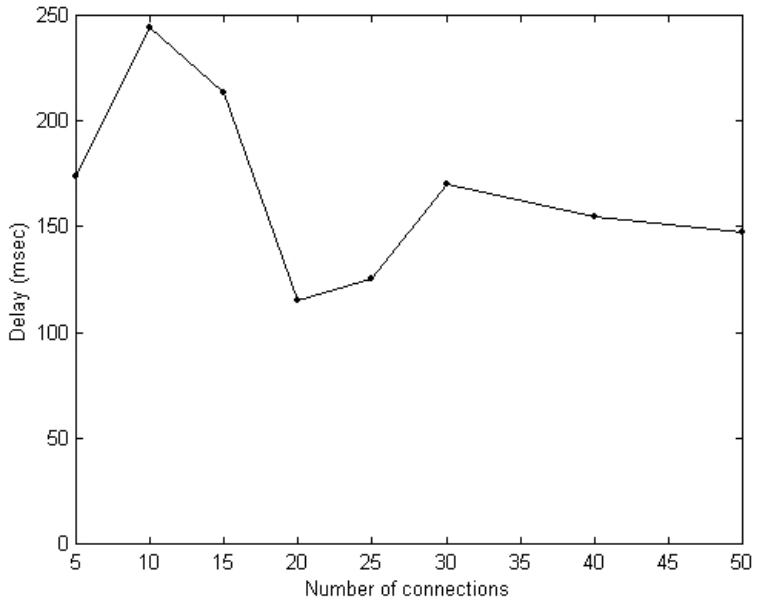

b. Transmission delay

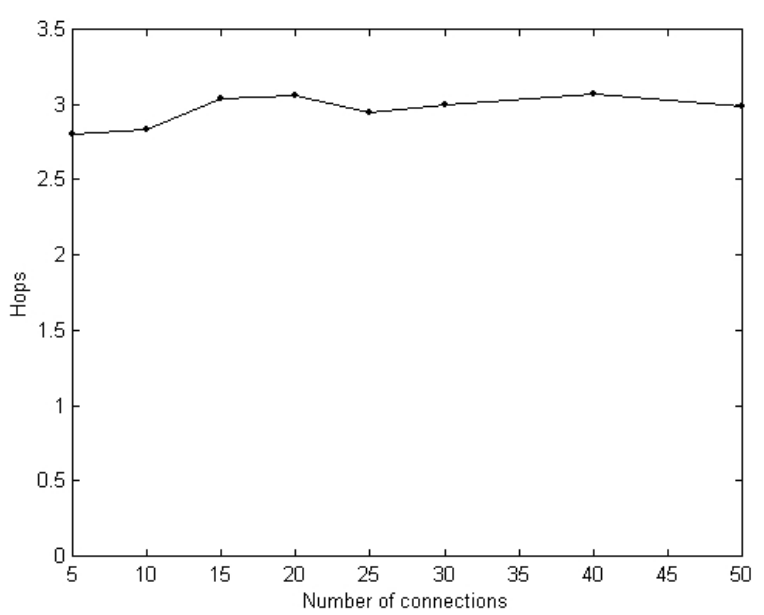

d. Hop count

Figure 8. Results of TCP data traffic 


\subsection{Real-time data traffic}

The real-time data traffic, such as voice and video, is dramatically increasing in internet and mobile wireless networks. It is characterized as transmitting a large volume of data in a time sensitive fashion. Due to the limited bandwidth and the error-prone link in wireless networks, it is very important to test if a MANET can support such kind of real-time data traffics.

We have applied the VoIP and video data traffics to various simulation scenarios. In the same scenario as that for CBR and TCP, it has been observed that video traffic performs poor. Acceptable performance can only be obtained when the number of connections is less than 10 . This is mainly due to the bandwidth limit, high application data rate, and the mobility in MANETs.

Figure 9 shows the simulation results of VoIP and Video when the number of connections is varying between 1 and 8 . The figure shows that almost all VoIP packets can be successfully received in a timely way. The delivery ratio only drops a little for higher number of connections. But for video traffic, when the number of connections is more than 3, the delivery ratio drops significantly from $99 \%$ to $50 \%$, and the transmission delay increases from $45 \mathrm{~ms}$ to $760 \mathrm{~ms}$. Two reasons for why the performance of video transmission for higher loads is not as good as VoIP are that: 1) VoIP is a particular case of CBR traffic, while the packet size of video traffic is varying in a large range as shown in Table 3. 2) The actual transmitted video data is much larger than that of VoIP, as shown in Figure 9.b, which implies that more network interference and congestion are introduced by the video traffic. 


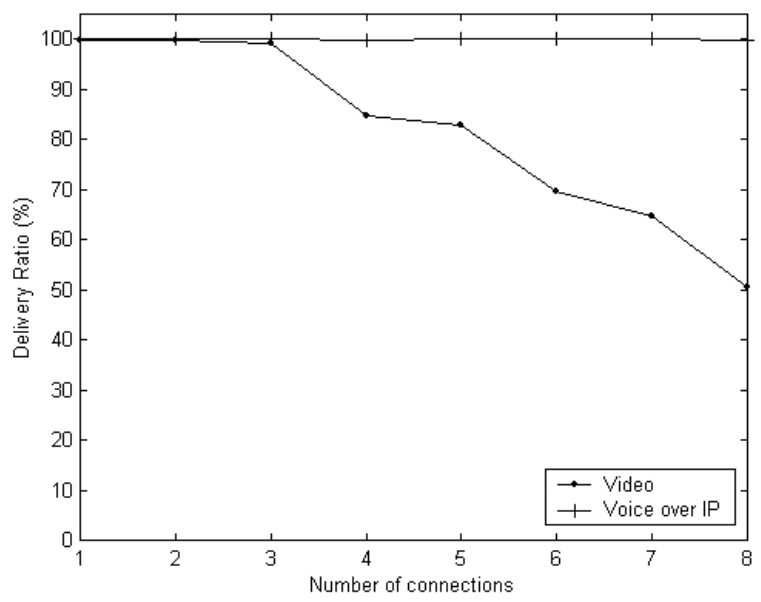

a. Delivery ratio

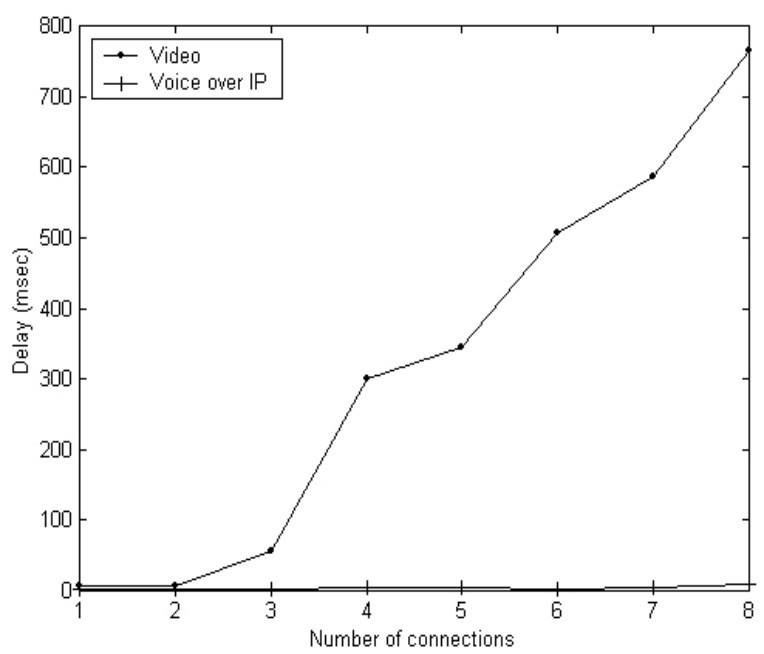

c. Transmission delay

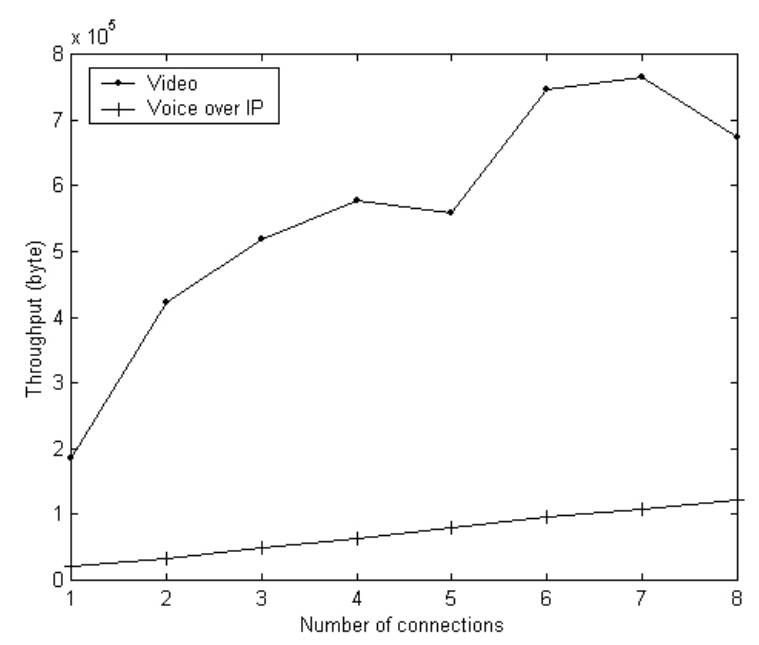

b. Throughput

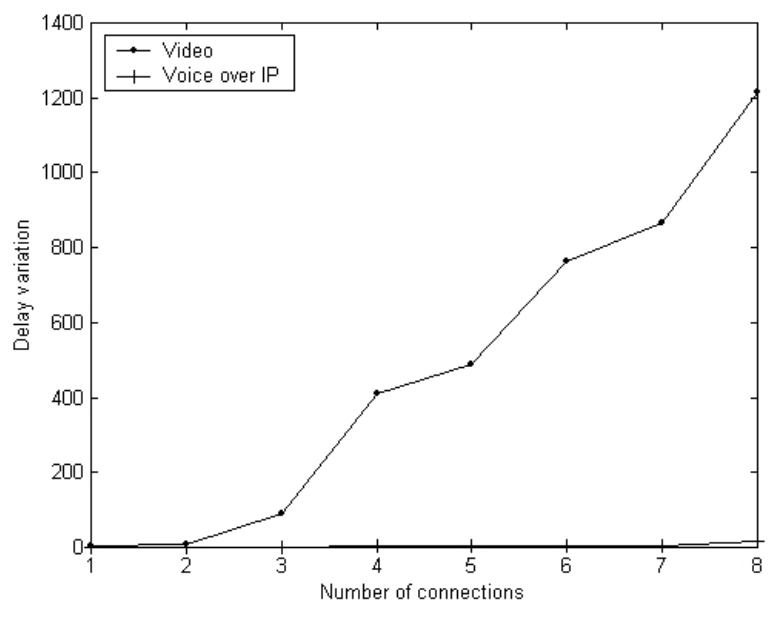

d. Delay variation (jitter)

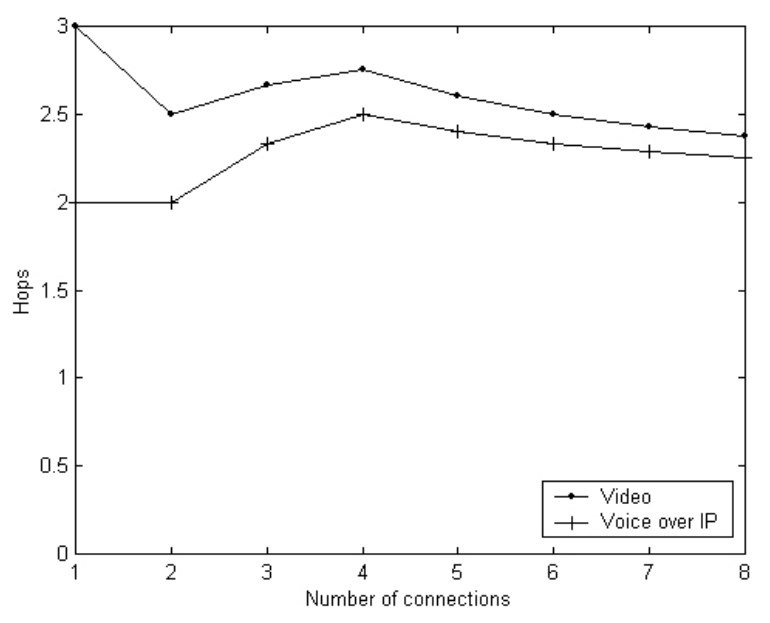

e. Hop count

Figure 9. Results of real-time data traffics 
One notation about the network transmission delay is that it is not equivalent to the time interval between packets. Instead, the network transmission delay only represents the time difference between the sending and receiving time of the same packet. For example, although the video packets are sent at interval of 0.04 second and the average network transmission delay for 5 connections is about 0.35 second, this does not necessarily mean that the quality of received video is violated. Actually, the $0.35-$ second delay may still be acceptable for most video applications in which some kind of buffer mechanism is employed so that a certain period of video is stored in advance before it is actually played back.

In most cases, throughput increases for higher loads because there are more data being sent and received. But since the packet size for video is varying in a large range, there might be the case when the throughput decreases while the number of packets increases, such as the 5-connection case in the Figure 9.b.

Figure 9.d displays the variation of transmission delay, or jitter, which is the standard deviation of transmission delay. It is shown that the video data transmission delay varies much more significantly for higher loads. This is because that more different data sending rates may be selected for different connections, depending on the level of network congestion.

\section{Simulation ON MOBILITY}

The mobility is an important character of MANETs. It can affect the system performance in various ways. This section studies the impact of two factors due to mobility: the position measurement error and the moving speed of mobile nodes.

\subsection{Position error}

We compare the system performance under different position measurement errors. The position 
error is randomly generated within the range of $0 \mathrm{~m}, \pm 25 \mathrm{~m}$, and $\pm 50 \mathrm{~m}$. The simulation results are given in Figure 10. It is shown that the system works pretty well for position errors within $\pm 25 \mathrm{~m}$, but $\pm 50 \mathrm{~m}$ position error does deteriorate the performance: the transmission delay significantly increases when the inaccuracy of position information increases.

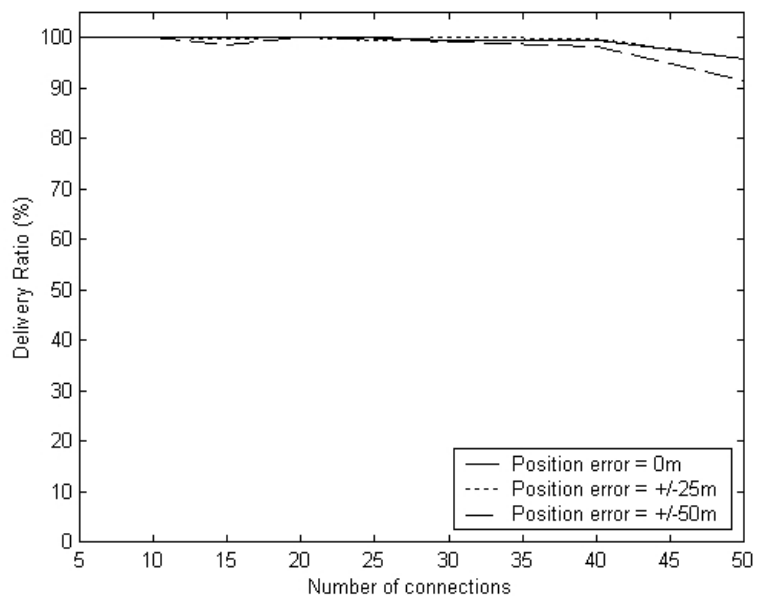

a. Delivery ratio

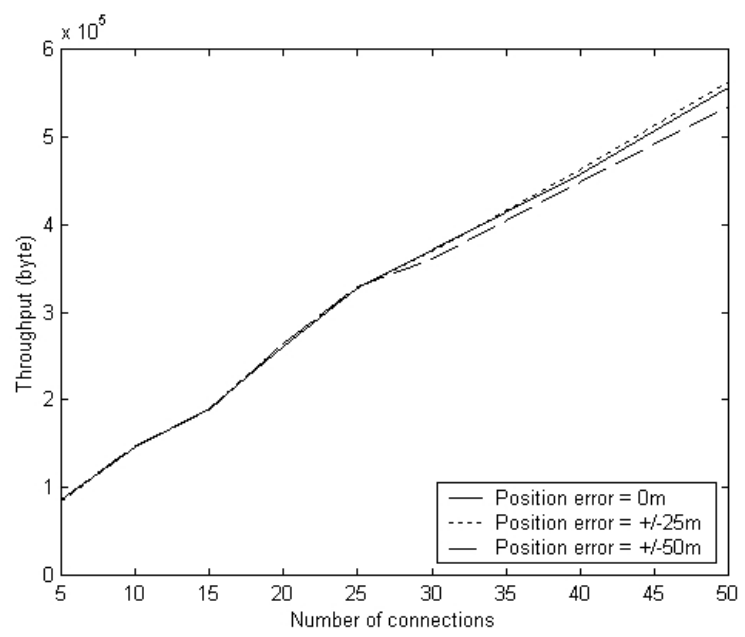

c. Throughput

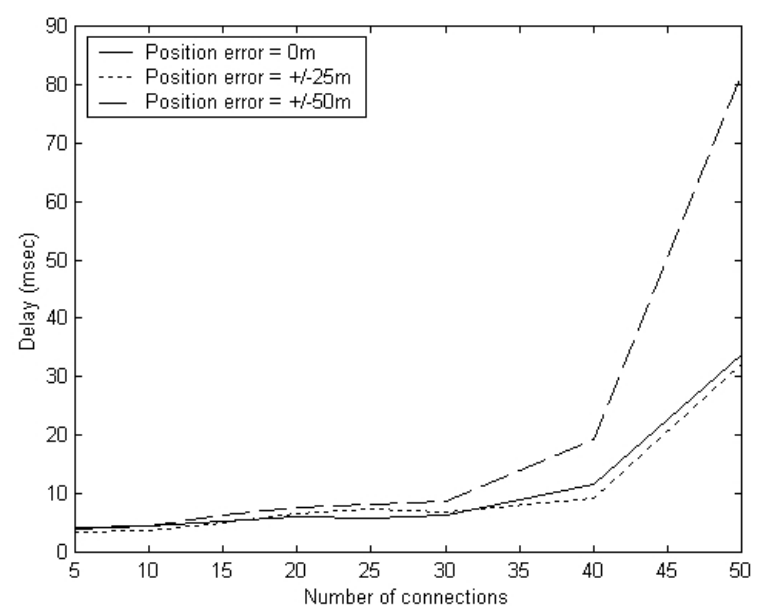

b. Transmission delay

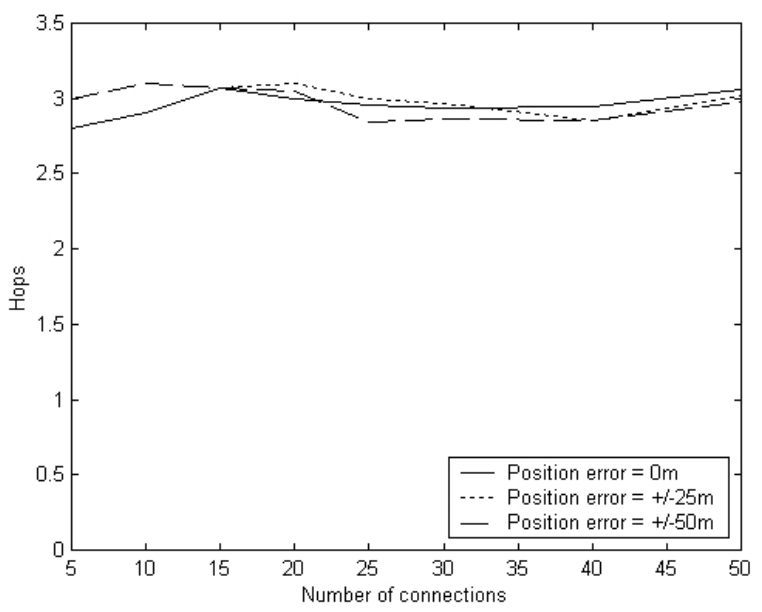

d. Hop count

Figure 10. Results of position error

\subsection{Moving speed}

The system performance is tested when mobile nodes are moving at different maximum speeds of $3 \mathrm{~m} / \mathrm{s}, 5 \mathrm{~m} / \mathrm{s}$, and $10 \mathrm{~m} / \mathrm{s}$. The update rate of position information is not fixed; it is updated whenever necessary in the routing protocol. It is shown in Figure 11 that the system works well for all these 
speeds. So when the maximal moving speed is less than $10 \mathrm{~m} / \mathrm{s}$, different moving speed does not introduce significant change of the delivery ratio, transmission delay, throughput, and hop count.

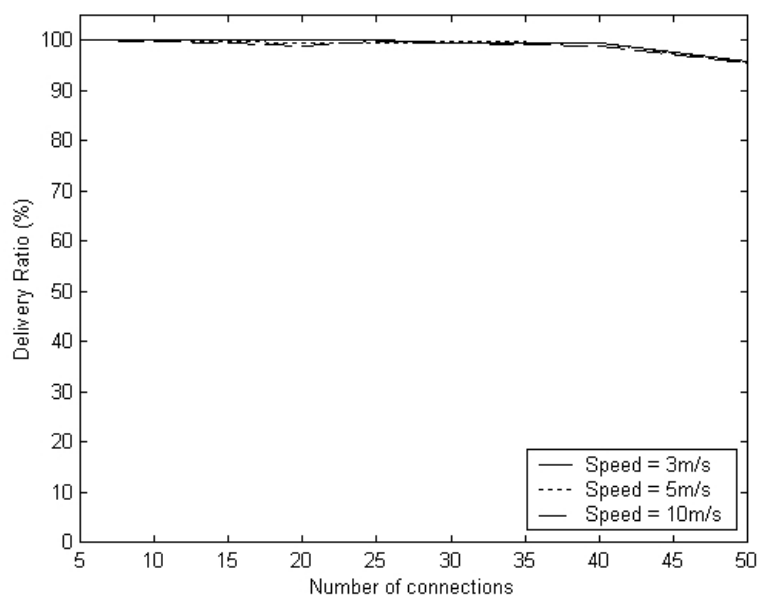

a. Delivery ratio

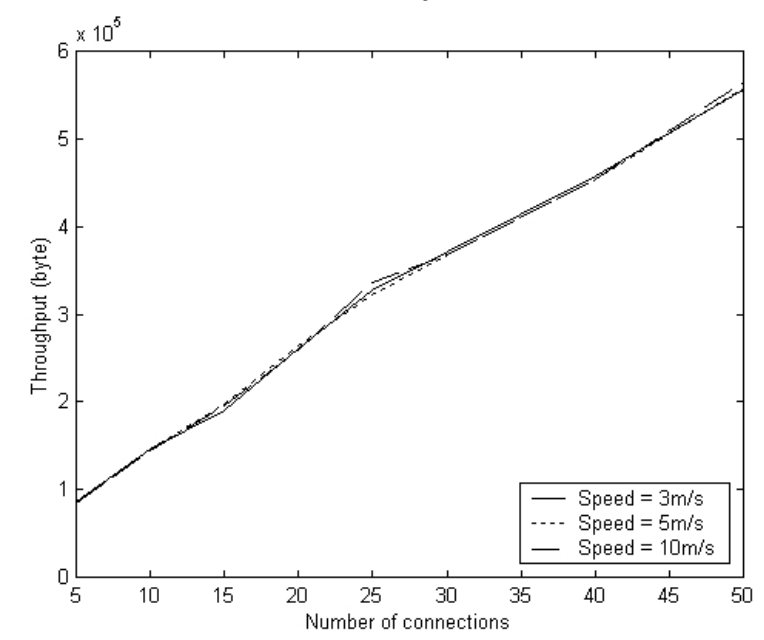

c. Throughput

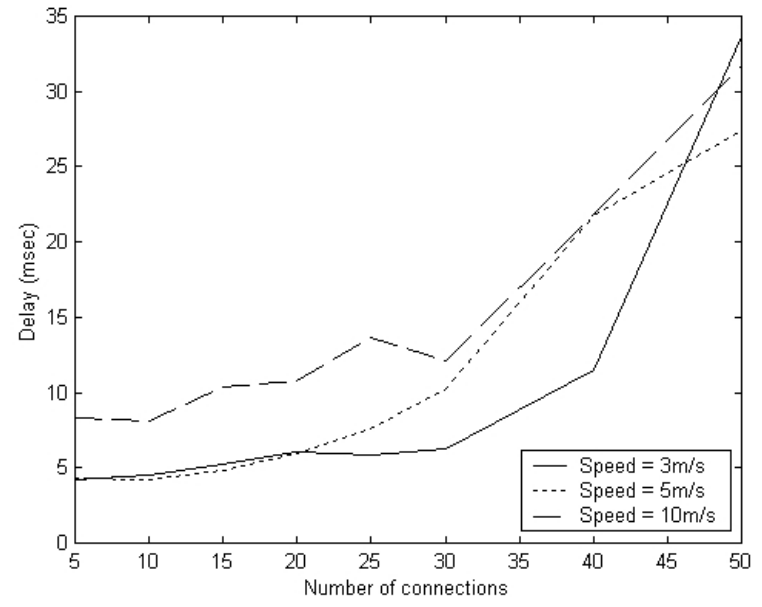

b. Transmission delay

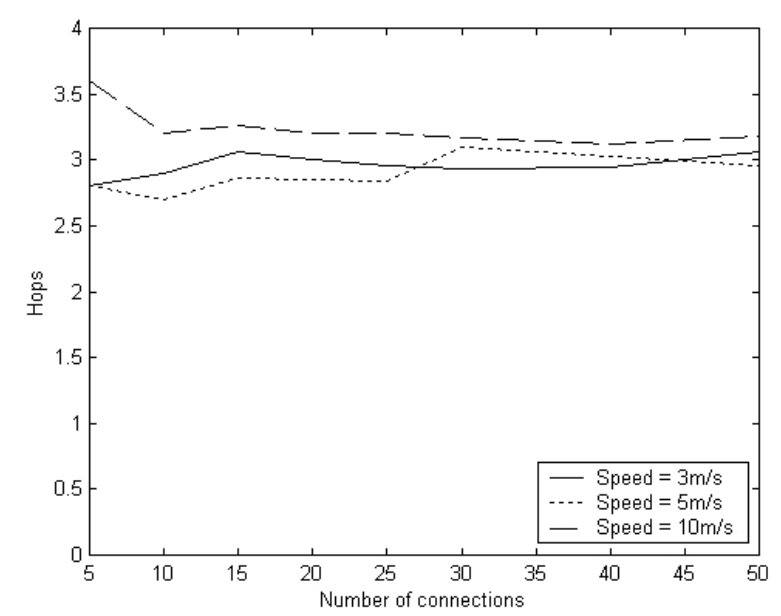

d. Hop count

\section{Figure 11. Results of mobility}

\section{Conclusion}

This paper proposes a simple link rate control algorithm for IEEE 802.11 based multiple-rate

MANETs. Extensive simulations are conducted to test the impact of various system components.

Several conclusions are drawn as follows.

It is found that the NAV should be estimated in RTS by small or the last used transmission rate. 
The update of NAV in CTS is preferred when the network load is low.

The carrier sense threshold should be no less than $70 \mathrm{dBm}$. But when it is large enough, larger threshold does not significantly improve the system performance.

CBR traffic performs very well. TCP traffic can provide similar delivery ratio to that of CBR, but it introduces longer transmission delay. VoIP traffic performs much better than video traffic. But neither VoIP nor video works well when the network load is higher than 10 connections. So the real-time data transmission over MANETs deserves further studies.

The large range of position error due to mobility may introduce longer transmission delay. The moving speeds less than $10 \mathrm{~m} / \mathrm{s}$ do not introduce significant impact on the system performance.

A general conclusion is that simulations have shown that many kinds of system components contribute to the overall performance of a MANET.

A last note about the above conclusions is that, since they depend on the given system parameters and scenarios, the exact number may not apply to the real system. For example, the $70 \mathrm{dBm}$ for carrier sense threshold may not necessarily be the same for various environments; the system performance might be significantly affected when the moving speed is larger than $10 \mathrm{~m} / \mathrm{s}$, and so on.

\section{ACKNOWLEDGEMENT}

The authors would like to thank Dr. Jeff Bonta and his colleagues at Motorola Communications Research Labs for many valuable discussions, suggestions and comments, Dr. Yi Lu for implementing the routing protocol for this study, and Mr. Shan Lei for implementing an early version of WalfischIkagami model and rate control algorithm. 


\section{REFERENCES}

[1] D. Remondo and I. G. Niemegeers, "Ad Hoc Networking in Future Wireless Communications," Computer Communications, 26: 36-40, 2003.

[2] M. Abolhasan, T. Wysocki, and E. Eutkiewicz, "A Review of Routing Protocols for Mobile Ad Hoc", Ad Hoc Networks, 2: 1-22, 2004.

[3] E. M. Royer and T.-K. Toh, "A Review of Current Routing Protocols for Ad Hoc Mobile Wireless Networks," IEEE Personal Communication Magazine, 46-55, April, 1999.

[4] http://www.isi.edu/nsnam/ns

[5] http://www.monarch.cs.cmu.edu

[6] http://pcl.cs.ucla.edu/projects/glomosim

[7] http://www.scalable-networks.com

[8] http://www.opnet.com

[9] R. Barr, Z. J. Haas, and R. Van Renesse, "Scalable Wireless Ad Hoc Network Simulation", in Handbook on Theoretical and Algorithmic Aspects of Sensor, 291-311.

[10] IEEE Standard 802.11: Wireless LAN Medium Access Control (MAC) and Physical Layer (PHY) specifications, 1999.

[11] C.E. Perkins and P. Bhagwat, "Highly Dynamic Destination-Sequenced Distance Vector Routing (DSDV) for Mobile Computers”, in Proceedings of ACM SIGCOMM, 1994.

[12] C. E. Perkins and E. M. Royer, "Ad-hoc On-Demand Distance Vector Routing", in Proceedings of the 2nd IEEE Workshop on Mobile Computing Systems and Applications, 90-100, 1999.

[13] D. Johnson and D. Maltz, "Dynamic Source Routing in Ad Hoc Wireless Networks", in Proceedings of ACM SIGCOMM-Computer Communication Review, 1996.

[14] E. Damosso, "Digital Mobile Radio: COST 231 View on the Evolution towards 3rd Generation Systems," in Final Report of the COST 231 Project, 1998.

[15] G. Holland, N. Vaidya and P. Bahl, “A Rate-Adaptive MAC Protocol for Multi-Hop Wireless Networks", in Proceedings of ACM MobiCom, 2001. 
[16] IEEE Standard 802.11g: wireless LAN Medium Access Control (MAC) and Physical Layer (PHY) specifications, Further Higher Data Rate Extension in the $2.4 \mathrm{GHz}$ Band, 2003.

[17] B. Karp and H. T. Kung, "GPSR: Greedy Perimeters Stateless Routing for Wireless Network," in Proceedings of ACM MobiCom, 2000.

[18] Video Traces for Network Performance Evaluation, http://trace.eas.asu.edu.

[19] F. Fitzek and M. Reisslein, "MPEG-4 and H.263 Video Traces for Network Performance Evaluation," IEEE Network, 15(6): 40-54. 2001.

[20] X. Wu, G. Ding, and B. Bhargava, "Impact of Link Distance on End-to-End Throughput in Multi-Rate Ad Hoc Networks", submitted to IEEE Trans. Mobile Computing, 2005.

[21] T. S. Rappaport, Wireless Communications, Principles and Practice, Prentice Hall, 1996.

[22] R. J. Punnoose, P. V. Nikitin, and D. D. Stancil, "Efficient Simulation of Ricean Fading within a Packet Simulator," in Proceedings of Vehicular Technology Conference, 2000.

[23] J-M Dricot and P. De Doncker, "High-accuracy Physical Layer Model for Wireless Network Simulations in ns-2," in Proceedings of International Workshop on Wireless Ad-Hoc Networks, 2004.

[24] D. Qiao, S. Choi, and K. G. Shin, "Goodput Analysis and Link Adaptation for IEEE 802.11a Wireless LANs," IEEE Trans. on Mobile Computing, 1(4): 278-292, 2002.

[25] J. Del P. Pavon and S. Choi, "Link Adaptation Strategy for IEEE 802.11 WLAN via Received Signal Strength Measurement," in Proceedings of ICC, 2003.

[26] O. Queseth, F. Gessler, and M. Frodigh, "Algorithms for Link Adaptation in GPRS," in Proceedings of IEEE VTC, 1999.

[27] Z. Lin, G. Malmgren, and J. Torsner, "System Performance Analysis of Link Adaptation in HiperLAN Type 2," in Proceedings of IEEE VTC, 2000.

[28] G. K. Chan, "Propagation and Coverage Prediction for Cellular Radio Systems," IEEE Transactions on Vehicular Technology, 40(4): 665-670, 1991.

[29] S. K. Kandasamy1, M. Ismail1, and S. K. Tiong, "Application of Genetic Algorithm for Large Scale Coverage Prediction in GSM Cellular Mobile System," International Symposium on Information and Communications Technologies, 2004. 


\section{APPENDIX: WALFISCH-IKEGAMI MODEL}

The Walfisch-Ikegami model considers the blocks between the transmitter and the receiver. The accuracy of this empirical model is quite high in urban environments where the propagation over the rooftops is significant. The model includes two cases, the Line Of Sight (LOS) and the None Line Of Sight (NLOS). For LOS case, the path loss is $L=42.6+26 \lg d_{k m}+20 \lg f_{M H z}$.

For the NLOS case, $L_{N L O S}=L_{0}+\max \left(L_{r t s}+L_{m s d}, 0\right)$,

where the free space loss $L_{0}=32.44+20 \lg d_{k m}+20 \lg f_{M H z}$, the rooftop-to-street diffraction loss

$$
L_{r t s}=-16.9-10 \lg w+10 \lg f_{M H z}+20 \lg \left(h_{\text {roof }}-h_{2}\right) \text {, }
$$

with the orientation loss

$$
L_{\text {Ori }}=\left\{\begin{array}{l}
-10+0.354 \varphi, \quad \text { for } 0^{\circ} \leq \varphi<35^{\circ} \\
2.5+0.075(\varphi-35), \quad \text { for } 35^{\circ} \leq \varphi<55^{\circ}, \\
4.0-0.114(\varphi-55), \quad \text { for } 55^{\circ} \leq \varphi<90^{\circ}
\end{array}\right.
$$

and the multiple screen diffraction loss

$$
L_{m s d}=L_{b s h}+k_{a}+k_{d} \lg d_{k m}+k_{f} \lg f_{M H z}-9 \lg b,
$$

with $L_{b s h}=-18 \lg \left(1+\max \left(h_{1}-h_{\text {roof }}, 0\right)\right)$,

$$
\begin{aligned}
& k_{a}=\left\{\begin{array}{l}
54, \quad \text { for } h_{1}>h_{\text {roof }} \\
54-0.8\left(h_{1}-h_{\text {roof }}\right), \quad \text { for } \quad d \geq 0.5 \mathrm{~km} \text { and } h_{1} \leq h_{\text {roof }} \\
54-0.8\left(h_{1}-h_{\text {roof }}\right) d_{k m} / 0.5, \quad \text { for } d<0.5 \mathrm{~km} \text { and } h_{1} \leq h_{\text {roof }}
\end{array}\right. \\
& k_{d}=18-15 \min \left(h_{1}-h_{\text {roof }}, 0\right),
\end{aligned}
$$

and $k_{f}=-4+\left\{\begin{array}{ll}0.7\left(f_{M H z} / 925-1\right), & \text { for medium sized city and suburban centers } \\ 1.5\left(f_{M H z} / 925-1\right), & \text { for metropolitan centers }\end{array}\right.$. 


\section{Biographies}

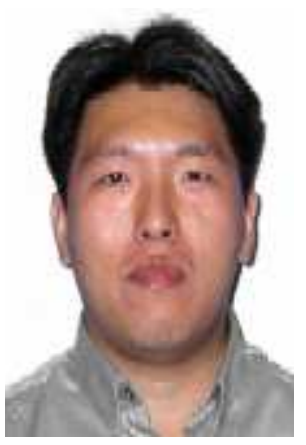

Gang Ding received his B.E. in control engineering from Tsinghua University, China. He is now a Ph.D. candidate in ECE Purdue University. Gang worked in Mitsubishi Electric Research Labs and Intel Research in 2004 and 2005. His research interests include mobile wireless communications and networking, distributed systems, and control theory.

Xiaoxin Wu received his B.E. degree from Beijing University of Posts and Telecommunications in 1990 and the Ph.D. degree from University of California, Davis in 2001. Since 2002, he has been working as a postdoctoral researcher in Department of Computer Science, Purdue University. He is currently supported by Institute for Information Infrastructure Protection (I3P) research Fellowship and working on wireless network privacy and security. His other research interests include designing and developing architecture, algorithm, and protocol for wireless network performance improvement. He will join Intel Labs at Beijing in 2006.

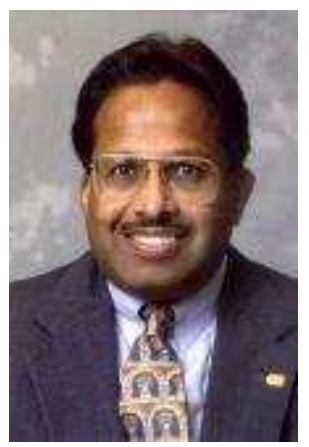

Bharat Bhargava received his B.E. from the Indiana Institute of Science and the M.S. and Ph.D. in EE from Purdue University. He is now a professor of computer sciences at Purdue University. His research involves mobile wireless networks, host authentication and key management, secure routing and dealing with malicious hosts, adaptability to attacks, and experimental studies. He has been awarded the charter Gold Core Member distinction by the IEEE Computer Society for his distinguished service. In 1999, he received an IEEE Technical Achievement award for a major impact of his decade long contributions to foundations of adaptability in communication and distributed systems. 